\title{
The Feasibility of Mechanical Thrombectomy on Single-Plane Angiosuite: An In-Depth Analysis of Procedure Time
}

\author{
Hiroyasu Inoue $^{a} \quad$ Masahiro Oomura $^{a}$ Yusuke Nishikawa ${ }^{b}$ Mitsuhito Mase ${ }^{b}$ \\ Noriyuki Matsukawa ${ }^{a}$ \\ aDepartment of Neurology, Nagoya City University Graduate School of Medical Sciences, Nagoya, Japan; \\ ${ }^{b}$ Department of Neurosurgery, Nagoya City University Graduate School of Medical Sciences, Nagoya, Japan
}

\section{Keywords}

Mechanical thrombectomy · Single plane - Biplane angiosuite $\cdot$ Guiding catheter placement $\cdot$ Acute ischemic stroke

\begin{abstract}
Introduction: Mechanical thrombectomy (MT) is usually performed on biplane (BP) angiosuites. When the BP angiosuite is not available, the single-plane (SP) angiosuite may be a substitute. However, the feasibility of MT performed on the SP angiosuite is yet to be elucidated. Therefore, we investigated the alternative effect of the SP angiosuite on the detailed division of procedure time, recanalization rate, and outcome in patients with anterior circulation infarction. Methods: The subjects included 80 consecutive patients with anterior circulation infarction who underwent MT at our hospital between May 2015 and December 2020. Demographics and characteristics of the BP and SP groups were assessed and compared. The time from puncture to guiding catheter placement ( $\mathrm{P}-\mathrm{G})$, time from guiding catheter placement to recanalization (G-R), and time from puncture to recanalization ( $\mathrm{P}-\mathrm{R}$ ) were also extracted. A good outcome was defined as a modified Rankin scale score $\leq 2$ at 3 months. Results: Of the 80 patients, 67 and 13 were treated
\end{abstract}

karger@karger.com www.karger.com/cee

Karger $\stackrel{\text { ' }}{5}$

GOPEN ACCESS
(C) 2021 The Author(s).

Published by S. Karger AG, Basel

This is an Open Access article licensed under the Creative Commons Attribution-NonCommercial-4.0 International License (CC BY-NC) (http://www.karger.com/Services/OpenAccessLicense), applicable to the online version of the article only. Usage and distribution for commercial purposes requires written permission. with BP and SP angiosuites, respectively. There were no differences in age, sex, complications, Alberta Stroke Program Early CT Score, National Institutes of Health Stroke Scale score at onset, occlusion site, rate of recombinant tissuetype plasminogen activator administration, stroke subtype, recanalization rate, and complications between the 2 groups. The rate of a good outcome was not different between the 2 groups. P-G was significantly longer in the SP group than in the BP group, whereas there was no significant difference in G-R and P-R between the 2 groups (P-G: BP $29.9 \pm 21.8$ vs. SP $48.5 \pm 43.6 \mathrm{~min}, p=0.04)$. Conclusion: MT performed on the SP angiosuite tended to prolong the time for guiding catheter placement. However, there was no difference in the overall procedure time, recanalization rate, or outcome between BP and SP angiosuites. Therefore, if the BP angiosuite is not available, the use of the SP angiosuite should be encouraged.

(c) 2021 The Author(s)

Published by S. Karger AG, Basel

\section{Introduction}

The efficacy of mechanical thrombectomy (MT) has been established [1-7], and patients with acute ischemic stroke (AIS) harboring large vessel occlusion should be 
promptly treated with MT [8]. As there is no elective MT procedure, healthcare facilities are not always prepared to perform MT.

Endovascular procedures for the brain are usually performed on a biplane (BP) angiography machine, where simultaneous opacification of a vessel from different views is obtained. As MT is an emergent procedure, the $\mathrm{BP}$ angiosuite is always available, resulting in the reluctant use of the single-plane (SP) angiosuite.

Rapid guiding catheter placement is critical for MT [9]. Therefore, we hypothesized that the time for placement of the guiding catheter of MT on the SP angiosuite would be longer than that of MT on the $\mathrm{BP}$ angiosuite. To the best of our knowledge, there have been no studies examining the detailed division of MT procedure time in terms of the type of angiosuite. Herein, we studied the feasibility of MT performed on the SP angiosuite and performed an in-depth analysis of the procedure time.

\section{Materials and Methods}

There were 95 consecutive patients with AIS who were diagnosed with AIS and underwent MT between May 2015 and December 2020 at Nagoya City University Hospital. Of the 95 patients, 15 had posterior circulation infarction, all of whom were treated with the BP angiosuite. In terms of analyzing the detailed division of procedure time, we limited our study to 80 patients with anterior circulation infarction to reduce the variability of the procedure. We collected details of baseline demographics, type of angiography suite, vascular risk factors, stroke subtype and severity, type of aorta, Alberta Stroke Program Early CT Score (ASPECTS), occlusion site, recanalization rate, periprocedural complications, and prognosis. A significant recanalization was defined as equal to or greater than thrombolysis in cerebral infarction IIb. A good outcome was defined as a modified Rankin scale (mRS) score of $\leq 2$ at 90 days. Additionally, the time from puncture to guiding catheter placement (P-G), time from guiding catheter placement to recanalization (G-R), and time from puncture to recanalization (P-R) were extracted. The time of contrast imaging when the guiding catheter was first identified in the desired position was defined as the guiding catheter placement time, and the time of contrast imaging when the decision was made to end the procedure was defined as the recanalization time. The reasons for the use of the SP angiosuite were also investigated.

\section{Angiography Suites}

MT was performed either using a BP angiography machine (Philips AlluraXper FD 20/20; Philips, The Netherlands) or an SP machine (Philips AlluraXper FD 20; Philips, The Netherlands). Angiosuites were regularly calibrated and controlled according to national and international standards.

\section{Statistical Analyses}

For continuous variables, the Mann-Whitney U test was used. Fisher's exact test was used for the qualitative variables. The sig-

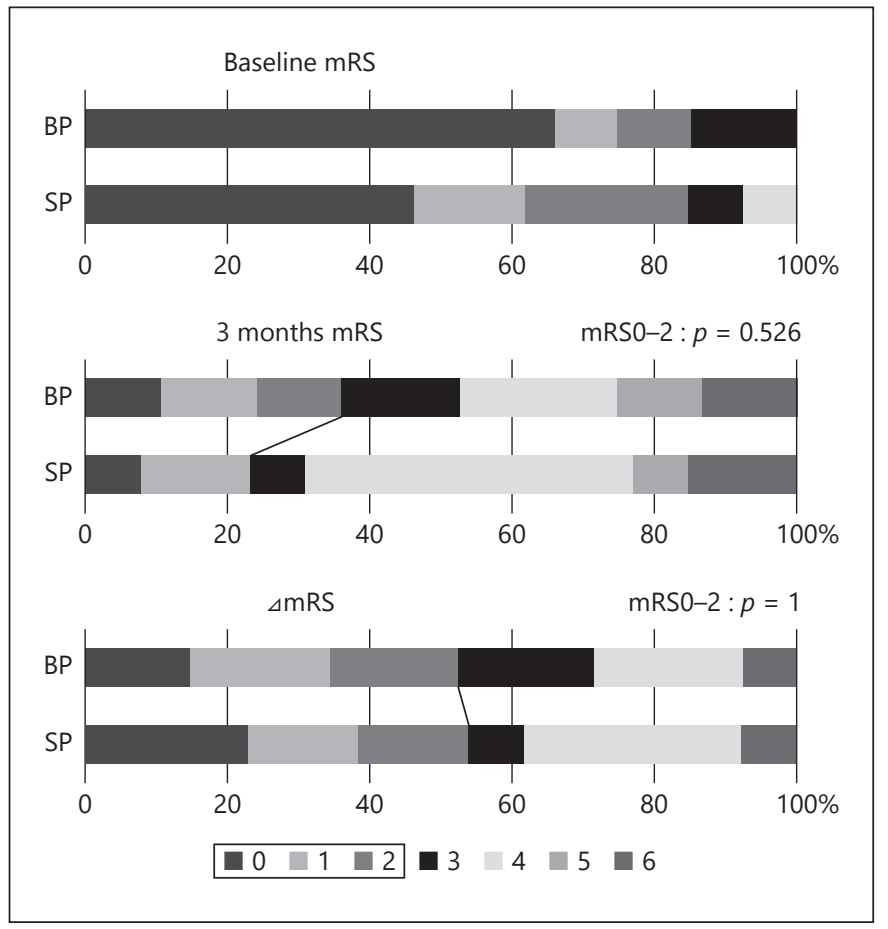

Fig. 1. Comparison of $\mathrm{mRS}$. There was no difference between the 2 groups in terms of good outcome (mRS0-2) after 3 months. There was no difference in $\triangle \mathrm{mRS}$. mRS, modified Rankin scale; $\triangle \mathrm{mRS}$, difference between baseline mRS and mRS after 3 months; $\mathrm{SP}$, single plane; BP, biplane.

nificance level ( $p$ value) was set at 0.05 . All statistical analyses were performed using EZR (Saitama Medical Center, Jichi Medical University, Saitama, Japan), which is a graphical user interface for R (The R Foundation for Statistical Computing, Vienna, Austria). More precisely, it is a modified version of $\mathrm{R}$ commander designed to add statistical functions frequently used in biostatistics.

\section{Results}

Of the 80 patients, 13 and 67 were treated with SP and $\mathrm{BP}$ angiosuites, respectively. The reasons for SP angiosuite use included the preoccupation of the BP angiosuite with scheduled procedure in 10 (62\%), emergency endovascular treatment in $2(15 \%)$, and machine maintenance in $1(8 \%)$ patient. There were no significant differences in age, sex, comorbidity, ASPECTS, occlusion site, type of aorta, National Institutes of Health Stroke Scale (NIHSS) score at onset, rate of recombinant tissue-type plasminogen activator administration, periprocedural complications, and recanalization rate between the 2 groups ( $\mathrm{Ta}$ ble 1). 
Table 1. Description of the single-plane and biplane groups

\begin{tabular}{|c|c|c|c|}
\hline $\begin{array}{l}\text { Parameters, } \\
\text { Mean } \pm \text { SD or } N(\%)\end{array}$ & $\begin{array}{l}\text { Single plane } \\
(N=13)\end{array}$ & $\begin{array}{l}\text { Biplane } \\
(N=67)\end{array}$ & \\
\hline Age & $75.15 \pm 14.7$ & $76.5 \pm 12.0$ & $p=0.97$ \\
\hline Sex M:F & $10: 3$ & $36: 31$ & $p=0.14$ \\
\hline Hypertension & $8(61.5)$ & $42(62.6)$ & $p=1$ \\
\hline Diabetes mellitus & $3(23.1)$ & $11(16.4)$ & $p=0.69$ \\
\hline Dyslipidemia & $5(38.5)$ & $24(35.8)$ & $p=1$ \\
\hline ASPECTS-DWI & $7.00 \pm 1.82(N=7)$ & $7.63 \pm 1.30(N=19)$ & $p=0.66$ \\
\hline ASPECTS CT & $7.85 \pm 1.77(N=7)$ & $8.64 \pm 1.96(N=51)$ & $p=0.11$ \\
\hline Onset NIHSS & $14.7 \pm 4.23$ & $18.5 \pm 6.49$ & $p=0.06$ \\
\hline IV rtPA & $6(46.2)$ & $41(61.2)$ & $p=0.37$ \\
\hline \multicolumn{4}{|l|}{ Aorta type } \\
\hline I & $4(30.8)$ & $21(31.3)$ & \\
\hline II & $4(30.8)$ & $26(38.8)$ & \\
\hline III & $5(38.5)$ & $20(29.9)$ & $p=0.93$ \\
\hline \multicolumn{4}{|l|}{ Stroke etiology } \\
\hline CES & $6(46.2)$ & $43(64.2)$ & \\
\hline ATBI & $2(15.4)$ & $12(17.9)$ & \\
\hline ESUS & $1(7.7)$ & $8(11.9)$ & \\
\hline Others & $4(30.8)$ & $4(6.0)$ & $p=0.075$ \\
\hline \multicolumn{4}{|l|}{ Vessel of occlusion } \\
\hline ACA & $0(0)$ & $2(3.0)$ & $p=0.40$ \\
\hline Ex ICA & $5(38.5)$ & $17(25.4)$ & \\
\hline In ICA & $0(0)$ & $12(18.0)$ & \\
\hline M1 & $5(38.5)$ & $23(34.3)$ & \\
\hline $\mathrm{M} 2$ & $5(38.5)$ & $20(29.9)$ & \\
\hline Tandem & $2(15.4)$ & $6(9.0)$ & $p=0.88$ \\
\hline \multicolumn{4}{|l|}{ Recanalization rate (TICI) } \\
\hline $0-2 a$ & $2(15.4)$ & $10(14.9)$ & \\
\hline $2 b-3$ & $11(84.6)$ & $57(85.1)$ & $p=1$ \\
\hline \multicolumn{4}{|c|}{ Duration of procedure, $\min$} \\
\hline P-G & $48.5 \pm 43.6$ & $29.9 \pm 21.8$ & ${ }^{*} p=0.04$ \\
\hline G-R & $61.8 \pm 35.0$ & $70.2 \pm 66.3$ & $p=0.87$ \\
\hline P-R & $110.4 \pm 54.7$ & $100.0 \pm 72.4$ & $p=0.30$ \\
\hline \multicolumn{4}{|l|}{ Complications } \\
\hline No & $9(69.2)$ & $46(68.7)$ & $p=0.641$ \\
\hline Asymptomatic ICH & $4(30.8)$ & $15(22.4)$ & \\
\hline Symptomatic ICH & $0(0)$ & $6(9.0)$ & \\
\hline
\end{tabular}

ASPECTS, Alberta Stroke Program Early CT Score; ATBI, atherothrombotic brain infarction; CES, cardioembolic stroke; ESUS, embolic stroke of undetermined sources; G, guiding; $\mathrm{ICH}$, intracranial hemorrhage; NIHSS, National Institutes of Health Stroke Scale; $\mathrm{P}$, puncture; $\mathrm{R}$, recanalization; rtPA, recombinant tissue-type plasminogen activator. * Significant between groups, $p<0.05$.
Although not significant, the mRS score before stroke onset tended to be slightly higher in the SP group than in the BP group $(p=0.10)$. However, there was no significant difference in a good outcome: BP $35.8 \%$ versus SP $23.1 \%(p=0.53)$. In the comparison of $\Delta \mathrm{mRS}$, which was defined as the difference between mRS at 3 months and $\mathrm{mRS}$ before onset, $\triangle \mathrm{mRS} 0-2$ was not significantly different between the 2 groups: $52.2 \%$ for BP and $53.8 \%$ for SP $(p=1.00)$ (Fig. 1).
There were no differences in G-R and P-R between the 2 groups (G-R min: BP $70.2 \pm 66.3$ vs. SP $61.8 \pm 35.0[p=$ 0.87 ], P-R min: BP $100.0 \pm 72.4$ vs. SP $110.4 \pm 54.7$ [ $p=$ $0.30])$. However, $\mathrm{P}-\mathrm{G}$ was significantly longer in the $\mathrm{SP}$ group than in the BP group (P-G min: BP $29.9 \pm 21.8$ vs. SP $48.5 \pm 43.6[p=0.04]$ ) (Fig. 2). There was no significant difference between the 2 groups in terms of complications (asymptomatic intracranial hemorrhage: BP 15 [22.4\%] vs. SP 4 [30.8\%]; symptomatic intracranial hemorrhage: BP 6 [9\%] vs. SP 0 [0\%] [ $p=0.641])$. 


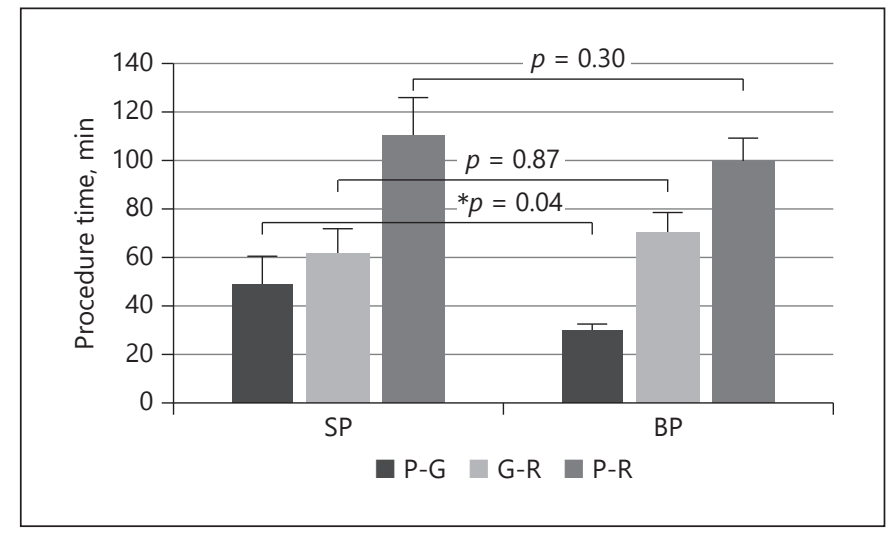

Fig. 2. Comparison of procedure time. $P-G$ was significantly longer in the SP group than in the BP group. G-R and P-R were not different between the 2 groups (Mann-Whitney $U$ test). P-G, puncture to guiding catheter placement; G-R, guiding catheter placement to recanalization; P-R, puncture to recanalization; SP, single plane; BP, biplane.

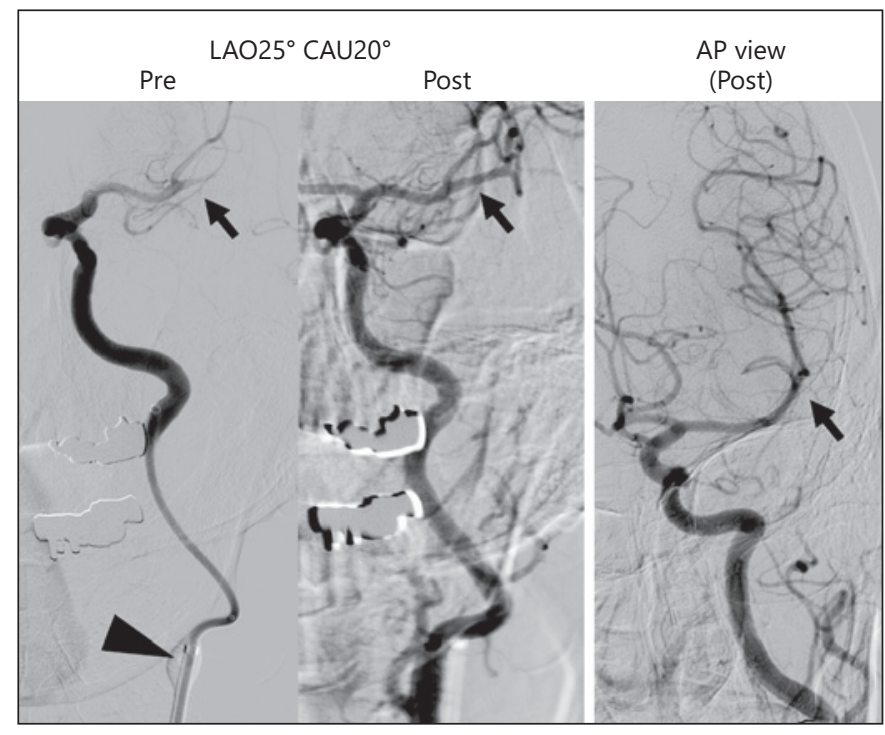

Fig. 3. Ingenuity of performing mechanical thrombectomy on a single-plane angiosuite. In the anteroposterior view, the M2 branches are overlapped and are difficult to delineate. By tilting the $\mathrm{X}$-ray projection slightly toward the affected side, the delineation of the branches becomes clearer (arrow). The tip of the guiding catheter is within the imaging range (black arrowhead), and treatment is performed using the image enlargement function as appropriate.

\section{Illustrative Case}

An 84-year-old woman developed AIS. She had persistent atrial fibrillation but was not treated with anticoagulants. She had dementia, knee osteoarthritis, and a premorbid mRS score of 3 . On admission, she had right hemiplegia, ocular deviation, and an NIHSS score of 17. Magnetic resonance (MR) diffusion-weighted imaging revealed a subtle ischemic change; her MRI-ASPECTS was 8 . Since MR angiography revealed an occlusion of the left M2 segment, she received endovascular treatment. Intravenous infusion of alteplase was not indicated because of the time window. The BP angiosuite was preoccupied with another elective procedure, and the treatment was performed on the SP angiosuite. MT was performed using the right femoral approach. A direct aspiration first-pass technique (ADAPT) with 6Fr SOFIAFLOW Plus (MicroVention Terumo, Tustin, CA, USA) was performed, and complete recanalization was achieved by the first pass. The duration times were 35,10 , and 45 min for P-G, G-R, and P-R, respectively. It was difficult to clearly delineate the occlusive lesion due to the overlapping of vessels by a straight posterior-anterior view. The right oblique view was helpful in delineating the bifurcation (Fig. 3).

\section{Discussion}

This study showed that MT on the SP angiosuite is feasible and does not prolong P-R. However, P-G tended to be slightly longer in the SP angiosuite than in the BP angiosuite.

To the best of our knowledge, there have been a few reports describing the feasibility of neurointervention on the SP angiosuite [10,11]. Previous studies have reported that the performance of MT on the SP angiosuite is feasible, and there is no difference in the total procedure time between SP and BP angiosuites [10,11]. This study is the first to report an in-depth analysis of MT procedure time on the SP angiosuite. Our results showed that the duration of placement of the guide catheter in the SP group tended to be longer than that in the BP group. Generally, neurointervention is divided into the vascular access and treatment phases [12]. Compared with neurointerventions other than MT, such as for aneurysms and dural arteriovenous fistulas, MT is considered to be less technically difficult. A previous study has shown that a short P-G is related to a good outcome in patients with AIS [9]. Thus, $\mathrm{P}-\mathrm{G}$ is critical for achieving rapid recanalization $[9,13]$. Although the vascular access stage is a critical part of the MT procedure, few studies have investigated the detailed distribution of MT procedure time on the SP angiosuite. Generally, placement of a guiding catheter is difficult in relation to the severity of atherosclerotic changes [9]. In such tortuous arteries, the wire ex- 
change technique can be helpful. In fact, in both of the 2 patients in the SP group in which the P-G was particularly long, a wire exchange technique from the femoral artery was attempted and failed, resulting in an alternative approach: a brachial approach. The unavailability of simultaneous imaging of the cranial vessels and aortic arch could be attributable to the failure. Additionally, frontal images are useful when selecting aortic branches, and lateral images are useful when selecting carotid bifurcation when we manipulate a catheter. With the SP angiosuite, it is necessary to change the angle of the X-ray at each time, which should be partly attributable to the prolonged $\mathrm{P}-\mathrm{G}$ in the SP group.

The overall median P-G in this study was longer than that in a previous report (20 $\mathrm{min}$ vs. $26 \mathrm{~min}$ ) [9]. Ribo et al. [9] have studied the effect of difficult catheter access in patients with AIS on clinical outcomes and reported that it was associated with a worse clinical outcome. According to their study, variables, including older age (age $>75$ years), hyperlipidemia, hypertension, and left carotid catheterization, were associated with difficult access [9]. In this study, the percentage of subjects aged $\geq 75$ years was as high as $59 \%$. This can be partly attributed to the prolongation of $\mathrm{P}-\mathrm{G}$.

Compared with neurointerventions on the BP angiosuite, those on the SP angiosuite can have some drawbacks: difficulties in obtaining the three-dimensional recognition of a vessel, especially in the cavernous segment of the internal carotid artery and bifurcation of the middle cerebral artery at M2, and limitation of view. An oblique position, such as the Haughton view, can compensate for the drawbacks and is useful for selecting artery branches such as the middle cerebral artery M2 bifurcation [14]. In addition, for distal lesions, including M3, it can be difficult to place the tip of the microcatheter and guiding catheter in a single radiation view. In such circumstances, an intermediate catheter can represent the movement of the guiding catheter.

Since a good prognosis is more likely to be unproportionate to $\mathrm{P}-\mathrm{R}$ in patients with AIS harboring large vessel occlusion, the effort to shorten P-R should be a critical issue [15]. The drip and ship triage has been advocated for non-MT-capable facilities; however, there should be some delay when patients are transferred. Previous studies comparing direct transfer to a comprehensive stroke center for thrombectomy versus secondary transfer have reported no difference in outcome [16], whereas others have reported better outcomes for the direct transfer group $[17,18]$. Our study showed that there was no significant difference in $\mathrm{P}-\mathrm{R}$, recanalization rate, complications, and good out- come (mRS0-2) between SP and BP angiosuites. Therefore, clinicians should be encouraged to perform MT on SP instead of transferring patients if the BP angiosuite is not available. As it has not been conclusive that the $\mathrm{BP}$ angiosuite is absolutely necessary for MT and the SP angiosuite entails a lower cost of installing and maintaining equipment than the BP angiosuite [19], clinicians should not be hesitant to perform MT on the SP angiosuite.

\section{Limitations}

This was a retrospective study and not a prospective randomized controlled trial. Additionally, the MT procedure time is influenced by many factors, including the experience of the operator, device, and angiosuites. Therefore, to unify the conditions as much as possible, we decided to conduct a single-center study. In addition, for ethical reasons, $\mathrm{SP}$ cannot be used in preference to $\mathrm{BP}$, and as a result, the number of SP cases may not be sufficient.

Other variables have not been examined, including the amount of radiation exposure and contrast medium used and interoperator changes. In addition, patients with posterior circulation infarction were excluded. Further studies are necessary in the future.

\section{Conclusion}

In patients with anterior circulation infarction, MT on the SP angiosuite tended to take a slightly longer time to place the guiding catheter than that on the $\mathrm{BP}$ angiosuite. However, there were no significant differences in recanalization rate, outcome, or safety between SP and BP angiosuites. Therefore, MT can be sufficiently performed on the SP angiosuite when the BP angiosuite is not available.

\section{Statement of Ethics}

This study was conducted following the approval from the ethics review committee (No. 60-20-0163) of our institution, and informed consent was obtained in the form of opt-out on the website.

\section{Conflict of Interest Statement}

The authors declare that they have no conflicts of interest to disclose.

\section{Funding Sources}

This research received no specific grant from any funding agency in the public, commercial, or not-for-profit sectors. 


\section{Author Contributions}

H.I. analyzed the data and drafted the manuscript. M.O. participated in data analysis, reviewed the drafts, and pointed out the problems of the study. Y.N. participated in the study design and reviewed the manuscript. M.M. and N.M. planned the study, presented the direction to the conclusion, and finally checked the manuscript.

\section{References}

1 Berkhemer OA, Fransen PS, Beumer D, van den Berg LA, Lingsma HF, Yoo AJ, et al. A randomized trial of intraarterial treatment for acute ischemic stroke. N Engl J Med. 2015 Jan; 372(1):11-20.

2 Goyal M, Demchuk AM, Menon BK, Eesa M, Rempel JL, Thornton J, et al. Randomized assessment of rapid endovascular treatment of ischemic stroke. N Engl J Med. 2015 Mar; 372(11):1019-30

3 Campbell BC, Mitchell PJ, Kleinig TJ, Dewey HM, Churilov L, Yassi N, et al. Endovascular therapy for ischemic stroke with perfusionimaging selection. N Engl J Med. 2015 Mar; 372(11):1009-18.

4 Saver JL, Goyal M, Bonafe A, Diener HC, Levy EI, Pereira VM, et al. Stent-retriever thrombectomy after intravenous t-PA vs. t-PA alone in stroke. N Engl J Med. 2015 Jun;372(24): 2285-95.

5 Jovin TG, Chamorro A, Cobo E, de Miquel MA, Molina CA, Rovira A, et al. Thrombectomy within 8 hours after symptom onset in ischemic stroke. N Engl J Med. 2015 Jun; 372(24):2296-306.

6 Nogueira RG, Jadhav AP, Haussen DC, Bonafe A, Budzik RF, Bhuva P, et al. Thrombectomy 6 to 24 hours after stroke with a mismatch between deficit and infarct. N Engl J Med. 2018 Jan;378(1):11-21.

7 Albers GW, Marks MP, Kemp S, Christensen S, Tsai JP, Ortega-Gutierrez S, et al. Thrombectomy for stroke at 6 to 16 hours with selec-

\section{Data Availability Statement}

The data that support the findings of this study are available from the corresponding author, M.O., upon reasonable request. tion by perfusion imaging. N Engl J Med. 2018 Feb;378(8):708-18.

8 Saver JL, Goyal M, van der Lugt A, Menon BK, Majoie CB, Dippel DW, et al. Time to treatment with endovascular thrombectomy and outcomes from ischemic stroke: a meta-analysis. JAMA. 2016 Sep;316(12):1279-88.

9 Ribo M, Flores A, Rubiera M, Pagola J, Mendonca N, Rodriguez-Luna D, et al. Difficult catheter access to the occluded vessel during endovascular treatment of acute ischemic stroke is associated with worse clinical outcome. J Neurointerv Surg. 2013 May;5 Suppl 1:i70-3.

10 Friedrich B, Maegerlein C, Lobsien D, Mönch $S$, Berndt M, Hedderich D, et al. Endovascular stroke treatment on single-plane vs. Bi-plane angiography suites. Clin Neuroradiol. 2019 Jun;29(2):303-9.

11 Guenego A, Mosimann PJ, Wintermark M, Heit JJ, Zuber K, Dobrocky T, et al. Safety and effectiveness of neuro-thrombectomy on single compared to biplane angiography systems. Sci Rep. 2020 Mar;10(1):4470.

12 Harrigan MR, Deveikis JP. Chapter 4, General Considerations for Neurointerventional Procedures. In: Handbook of cerebrovascular disease and neurointerventional technique. 3rd ed. USA: Humana Press; 2018. p. 167-246.

13 Shibata T, Oomura M, Nishikawa Y, Mase M. Direct brachial approach for acute basilar artery occlusion: technical note and preliminary clinical experience. Neurointervention. 2020 Mar;15(1):31-6.

14 Haughton VM, Rosenbaum AE, Baker RA, Plaistowe RL. Lateral projections with inclined head for angiography of basal cerebral aneurysms. Radiology. 1975 Jul;116(1):220-2.

15 Saver JL, Goyal M, van der Lugt A, Menon BK, Majoie CB, Dippel DW, et al. Time to treatment with endovascular thrombectomy and outcomes from ischemic stroke: a meta-analysis. JAMA. 2016 Sep;316(12):1279-88.

16 Aghaebrahim A, Jadhav AP, Hanel R, Sauvageau E, Granja MF, Zhang Y, et al. Outcome in direct versus transfer patients in the DAWN controlled trial. Stroke. 2019 Aug; 50(8):2163-7.

17 Seker F, Bonekamp S, Rode S, Hyrenbach S, Bendszus M, Möhlenbruch MA. Direct admission vs. secondary transfer to a comprehensive stroke center for thrombectomy: retrospective analysis of a regional stroke registry with 2797 patients. Clin Neuroradiol. 2020 Dec;30(4):795-800.

18 Zhao W, Ma P, Chen J, Yue X. Direct admission versus secondary transfer for acute ischemic stroke patients treated with thrombectomy: a systematic review and meta-analysis. J Neurol. 2020.

19 Bellemare CA, Poder TG. Effectiveness of biplane angiography compared to monoplane angiography for vascular neuro-interventions: a systematic review of the literature. Clin Radiol. 2017 Jul;72(7):612.e1-e5. 